\title{
Primary thyroid leiomyosarcoma: A case report and literature review
}

\author{
ZHEN-YU ZOU ${ }^{1}$, NING NING ${ }^{2}$, SONG-YAN LI ${ }^{1}$, JIE LI $^{3}$, XIAO-HUI DU ${ }^{1}$ and RONG LI ${ }^{1}$ \\ ${ }^{1}$ Department of General Surgery, Chinese People's Liberation Army General Hospital, Beijing 100853; \\ ${ }^{2}$ Department of Gastrointestinal Surgery, Peking University International Hospital, Beijing 102206 \\ ${ }^{3}$ Department of Pathology, Chinese People's Liberation Army General Hospital, Beijing 100853, P.R. China
}

Received January 15, 2015; Accepted February 11, 2016

DOI: $10.3892 / \mathrm{ol} .2016 .4496$

\begin{abstract}
Primary thyroid leiomyosarcoma (LMS) is an extremely rare soft tissue cancer; only 22 cases have been reported in the literature to date. In the current study, the case of an 83-year-old male patient who presented with a neck mass that had grown rapidly over the previous 3 months is reported. The patient underwent thyroid lobectomy twice and two cycles of immunotherapy for the treatment of primary thyroid LMS; however, he succumbed to the disease 5 months after the second surgery. An accurate diagnosis of primary thyroid LMS is difficult, as the disease is often misdiagnosed as anaplastic carcinoma, and requires the combined assessment of clinical, imaging and pathological data. Diagnosis of the current patient with primary thyroid LMS and a comprehensive review of the relevant literature are presented herein.
\end{abstract}

\section{Introduction}

Primary thyroid leiomyosarcoma (LMS) is an extremely rare soft tissue cancer, with only 22 reported cases to date (1-19). LMS is commonly observed in the gastrointestinal tract, retroperitoneum and pelvis $(10,16)$; however, only $0.014 \%$ of LMS cases develop in the thyroid gland $(6,10,11,13,18,19)$, with an estimated 1-year survival rate of 5-10\% $(13,17)$. Primary thyroid LMS most commonly occurs in the elderly with a predilection for female patients, and typically presents as a painless, rapidly growing neck mass. Ultrasound, computed tomography (CT) and magnetic resonance imaging (MRI) may be used to diagnose thyroid tumors. A diagnosis of LMS is dependent on the presence of smooth muscle-actin (SMA), which may be identified by immunohistochemical staining. The standard primary

Correspondence to: Professor Rong Li or Professor Xiao-Hui Du, Department of General Surgery, Chinese People's Liberation Army General Hospital, 28 Fuxing Road, Beijing 100853, P.R. China

E-mail: lirong@medmail.com.cn

E-mail: duxiaohui301@sina.com

Key words: leiomyosarcoma, thyroid neoplasms, diagnosis, histopathology, therapy treatment for primary thyroid LMS is radical surgery. The long-term prognosis is poor and $\sim 50 \%$ of patients succumb to the disease within a short period of time after diagnosis $(10,14)$. The present study reports the case of an 83-year-old male patient diagnosed with primary thyroid LMS and the relevant literature is comprehensively reviewed.

\section{Case report}

In December 2013, 83-year-old male patient presented to the Chinese People's Liberation Army General Hospital (Beijing, China) with a neck mass that had grown rapidly in the 3 months prior to admission, as well as hoarseness and bucking, which had been apparent for 1 month. No evidence of dyspnea or dysphagia were identified. The patient's past medical history included a diagnosis of thyroid carcinosarcoma with a right thyroid lobectomy performed in June 2013 (6 months previously), as well as a diagnosis of prostate cancer, which was treated with prostatectomy and orchiectomy in May 2010 (3 years previously), and diabetes treated with insulin from August 2010 ( 2 years prior to admission). The patient reported no radiation exposure or any family history of cancer.

Palpation revealed a large, irregular, firm, tender and immovable tumor mass in the right anterior neck. The trachea was displaced to the left. The results of a serum thyroid function test were as follows: Free triiodothyronine, $5.39 \mathrm{pmol} / 1$ (normal range, 2.76-6.3 pmol/1); free thyroxine, $26.79 \mathrm{pmol} / 1$ (normal range, 10.42-24.32 pmol/1); and thyroid stimulating hormone, $0.084 \mathrm{mU} / 1$ (normal range, 0.35-5.5 mU/1). Laryngoscopy (ENF-V2; Olympus Corporation, Tokyo, Japan) revealed right vocal cord paralysis. Ultrasonography (iU22; Philips, Amsterdam, Holland) of the thyroid identified a $12.2 \times 10.1-\mathrm{cm}$, ill-defined hypoechoic mass arising from the right thyroid lobe. CT (uCT S-160; United Imaging, Shanghai, China) revealed an ill-defined, low-density mass replacing the right thyroid lobe that extended to the substernal area and compressed the esophagus. The trachea was widely displaced to the left lateral neck. Contrast-enhanced CT (Brilliance iCT 728306; Philips Medical Systems, Cleveland, OH, USA) showed inhomogeneous enhancement, necrosis and cystic degeneration within the tumor (Fig. 1). No evidence of distant metastases was identified.

Surgical exploration revealed a firm, irregular, encapsulated tumor measuring $13.5 \times 10 \times 5 \mathrm{~cm}$ in size that occupied the 
entire right thyroid lobe. Subsequently, a right thyroid lobectomy was performed. The tumor was dissected at the lower edge of the submaxillary gland and parotid gland on the surface of the trachea and thyroid cartilage. No lymph node metastasis was identified.

The resected specimen $(4-\mu \mathrm{m})$ was fixed in $10 \%$ buffered formalin (Sigma-Aldrich, St. Louis, MO, USA), processed and embedded in paraffin (Leica, Mannheim, Germany) using standard histological methods (19). Staining was visualized using an inverted microscope (TE2000-U; Nikon Corporation, Tokyo, Japan). Hematoxylin and eosin (Sigma-Aldrich) staining revealed interlacing fascicles of spindle tumor cells in the tumor (Fig. 2). Immunohistochemistry was performed using a standard avidin-biotin immunoperoxidase technique (19). The monoclonal mouse anti-human actin (clone, 1A4; cat. no. IR611; dilution, 1:100; Dako, Glostrup, Denmark) was used for SMA staining (Fig. 3). The monoclonal mouse anti-human vimentin (clone, V9; cat. no. AX074-YCD; dilution, 1:200; BioGenex, USA) was used for vimentin staining. The monoclonal mouse anti-human Ki-67 antigen (clone, MIB-1; cat. no. IR626; dilution, 1:200; Dako) was used for Ki-67 staining. The monoclonal mouse anti-human cytokeratin (clone, AE1/AE3; cat. no. IR620; dilution, 1:100; Dako) was used for cytokeratin staining (Fig. 4). Immunohistochemical staining was positive for SMA (Fig. 3), vimentin and Ki-67 (50-75\%), and negative for cytokeratin (Fig. 4). No epithelial differentiation was identified. The final diagnosis was primary thyroid LMS, and was determined according to the following guidelines: i) Morphologically similar to LMS, with the tissue comprised of spindle cells arranged in fascicles with elongated and centrally located cell nuclei; ii) the assessment of cellular atypia, necrosis and mitotic activity per high-powered field, which can differentiate LMS from benign tumors; and iii) the presence of muscle specific markers, including SMA positivity (20).

The patient received 2 cycles of adoptive transfer of immune cell therapy on January 2014 and February 2014. Peripheral blood mononuclear cells (PBMC) were isolated from the patient on the day prior to operation allowing time to prepare the immune cells. The patient was sequentially injected intradermally with autologous tumor lysate-loaded dendritic cells (DCs) $\left(>1 \times 10^{8}\right)$ at multiple areas in the clavicular region on day 13 post-PBMC collection, and infused intravenously with DC-activated $\mathrm{T}$ lymphocyte cells $\left(>1 \times 10^{10}\right)$ on day 14 post-PBMC collection. However, 2 months after the completion of immunotherapy, tumor recurrence without multiple metastases was identified in the right neck. The patient's general condition deteriorated gradually and he succumbed to the disease 5 months after the second surgery.

\section{Discussion}

The first case of LMS of the thyroid gland was reported in 1969; in this case, the tumor metastasized to the heart and brain (1). To date, only 22 cases of primary thyroid LMS have been reported in the literature (Table I) (1-19). However, the number of reported cases is increasing due to the utilization of immunohistochemical methods to identify SMA positivity for diagnosis (6).

Primary thyroid LMS most commonly occurs in elderly patients, with an average age of 65.3 years (range,
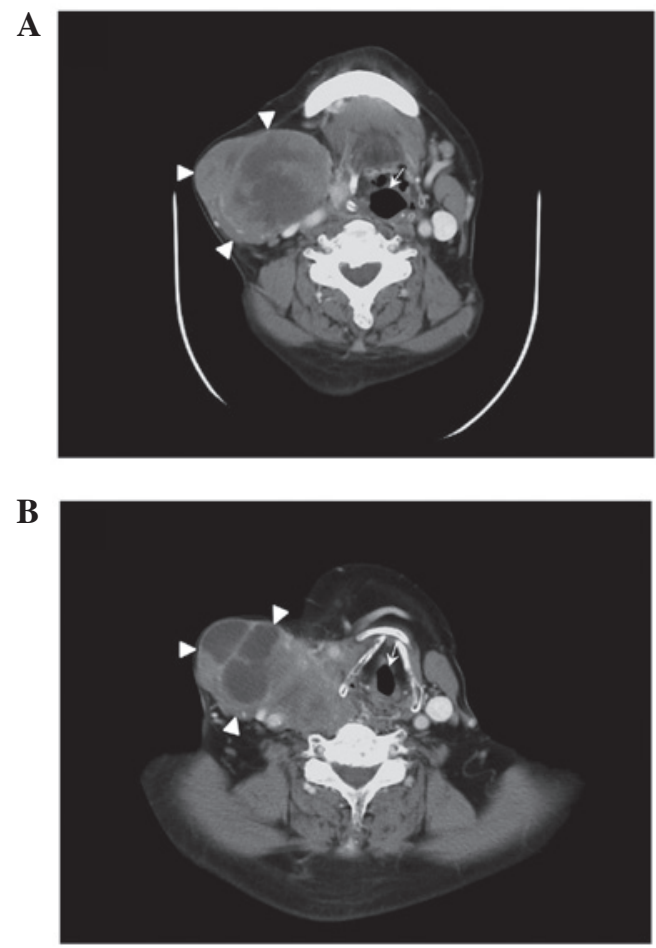

Figure 1. Contrast-enhanced computed tomography scan of the neck revealing inhomogeneous enhancement of a low-density mass (triangles), measuring $12 \mathrm{~cm}$ in diameter, in the right lobe of the thyroid gland. (A) Necrosis and (B) cystic degeneration were observed in the tumor. The trachea was widely displaced to the left side (arrowhead).

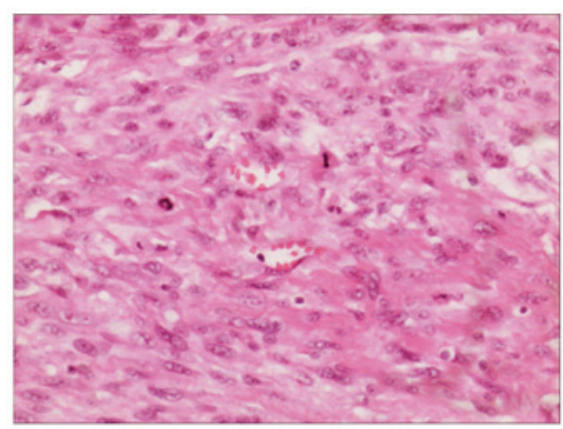

Figure 2. Histopathological examination revealing that the tumor was composed of interlacing fascicles or bundles of spindle cells with hyperchromatic, blunt nuclei and abundant eosinophilic cytoplasm (stain, hematoxylin and eosin; magnification, x200).

39-90 years). However, a case of Epstein-Barr virus-associated thyroid LMS was reported in a 6-year-old male patient with congenital immunodeficiency (9). Primary thyroid LMS exhibits a slight predilection for female patients (female:male ratio, 1.3:1). Primary thyroid LMS most commonly presents as a painless, rapidly growing neck mass. Additional symptoms include hoarseness, dysphagia, dyspnea and weight loss, and arm pain is occasionally reported (1,2,5-8,10,13-19). According to the literature, the majority of patients with LMS are euthyroid (2,4-6,11-14,15-19). Notably, none of the previous cases reported a history of radiation exposure. Tumor diameter varies between 1.9 and $16 \mathrm{~cm}$ (mean diameter, $6.6 \mathrm{~cm}$ ), and tumors are solitary, with the majority confined to a single lobe (4-7,9-19). 


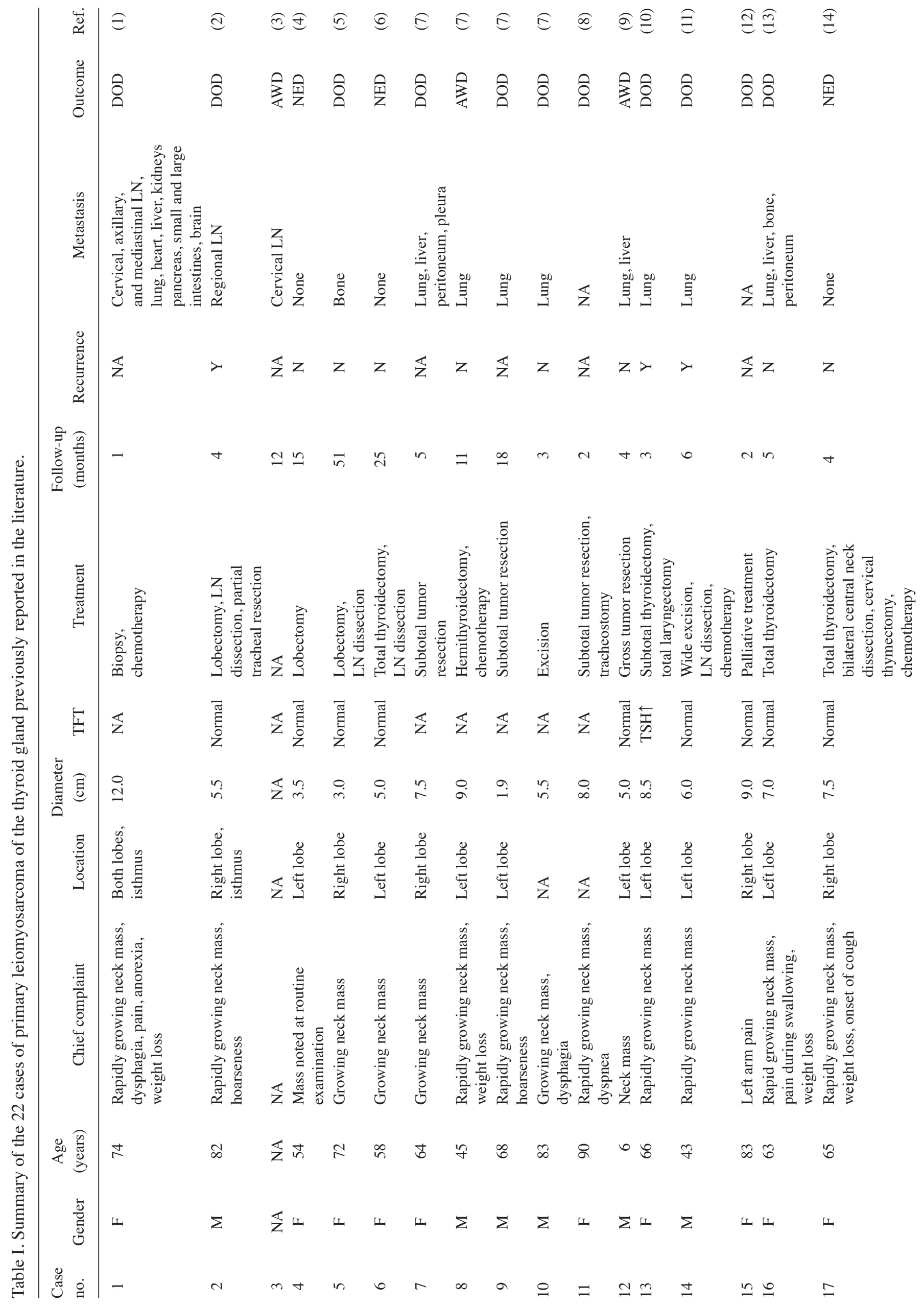




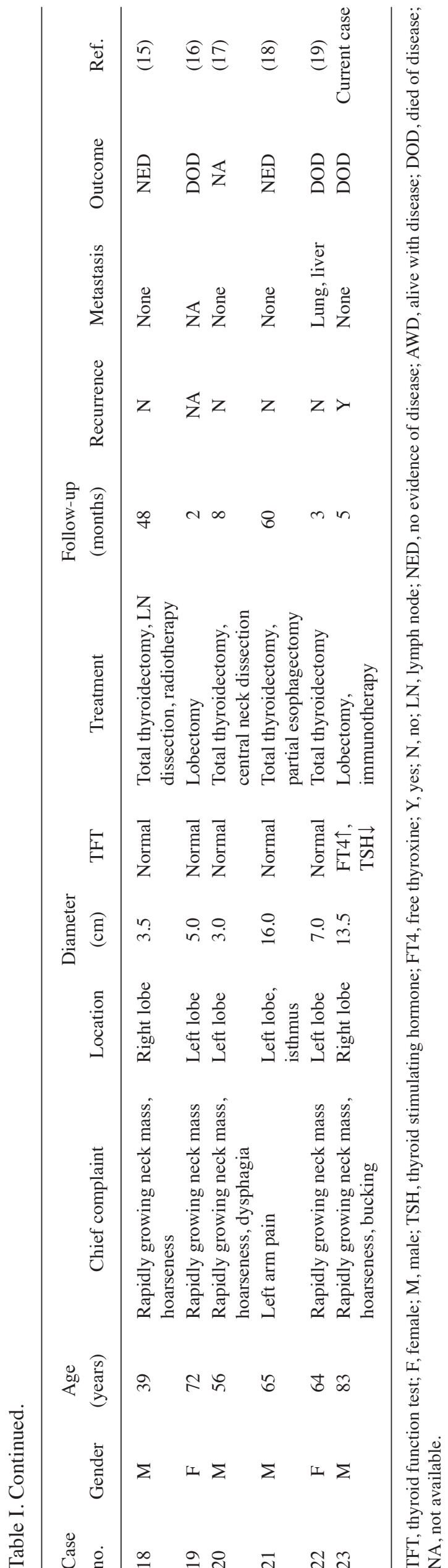

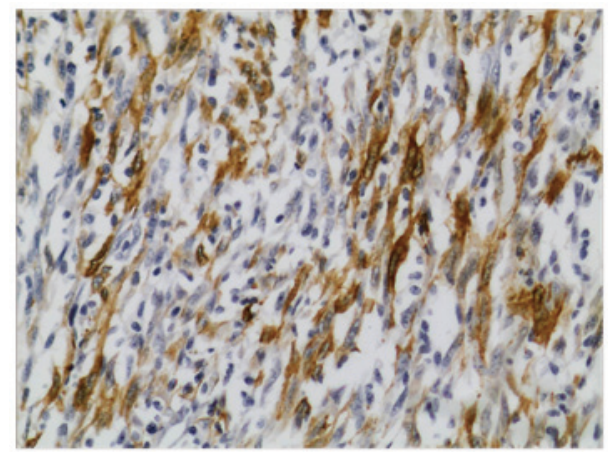

Figure 3. Immunohistochemical staining of tumor cells revealing positivity for smooth muscle actin (magnification, x200).

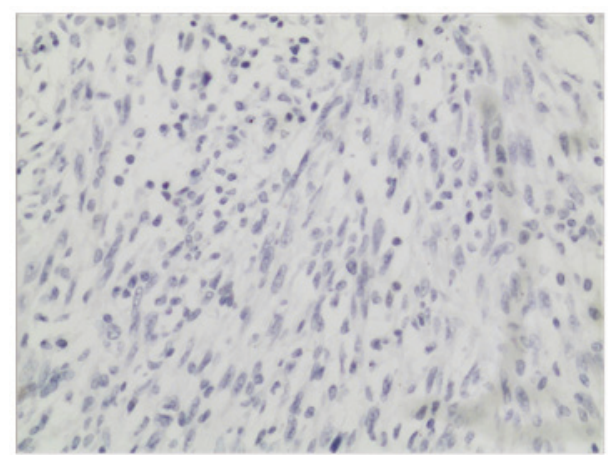

Figure 4. Immunohistochemical staining of tumor cells revealing negativity for cytokeratin (magnification, x200).

Imaging results may reveal a variety of characteristics regarding primary thyroid LMS. For example, thyroid scans may identify a cold nodule, or an enlarged gland with areas of increased or decreased uptake of radioactive iodine $(1,4,7)$; ultrasound may reveal an ill- or well-defined hypoechoic mass, a solid or partially cystic nodule, or a calcified nodule $(2,4-6,10)$; CT may show a low-density mass with dense calcification and necrosis, a well-demarcated mass, a soft tissue mass with calcification or, in certain cases, direct tumorous invasion of the adjacent structures $(5,6,10)$; and MRI may reveal a mass of intermediate signal on T2-weighted images and an isointense mass on T1-weighted images with a fair gadolinium enhancement (10).

Fine needle aspiration cytology is also used for the preoperative diagnosis of thyroid LMS (18). Histologically, the presence of interlacing fascicles or bundles of eosinophilic spindle cells and positivity for SMA on immunohistochemical examination may diagnose thyroid LMS $(10,16)$. LMS tumor cells in the thyroid and other organs typically stain positively for SMA, vimentin and desmin (19), and negatively for cytokeratin, thyroglobulin, calcitonin, S100 and chromogranin (16). Notably, negative staining for cytokeratin, a protein composed of keratin-containing intermediate filaments found in the intracytoplasmic cytoskeleton of epithelial tissue, indicates a non-epithelial tumor. Furthermore, c-kit is rarely expressed in LMS, although a case of c-kit overexpression in primary thyroid LMS has been previously reported (11).

In the present case, the clinical manifestation (a rapidly growing neck mass confined to a single lobe) and pathological 
features $\left(\mathrm{SMA}^{+}\right.$, vimentin ${ }^{+}, \mathrm{Ki}-67^{+}$and cytokeratin $\left.{ }^{-}\right)$were similar to those reported in the literature. However, the current patient was older than previous cases and had an abnormal thyroid function. The patient was initially misdiagnosed with thyroid carcinosarcoma prior to the first thyroidectomy as cytokeratin staining, which serves an important role in distinguishing epithelioma and non-epithelial tumors, was not taken into account.

The major histopathological differential diagnoses of primary thyroid LMS include anaplastic carcinoma of the thyroid, spindle cell variant of medullary thyroid carcinoma, spindle cell tumor with thymus-like differentiation, and uncommon primary and metastatic tumors of the thyroid with predominant spindle cells (19). The World Health Organization categorizes carcinosarcoma as a variant of anaplastic carcinoma. However, Agrawal et al (21) proposed that 'thyroid carcinosarcoma' should be considered a distinct entity. Unlike anaplastic carcinoma originating from epithelial cells, carcinosarcoma is hypothesized to originate from malignant epithelial (carcinomatous) and mesenchymal cells (21). Positive immunohistochemical staining for thyroglobulin (in carcinomatous cells), and vimentin and S100 (in mesenchymal cells) confirms a diagnosis of carcinosarcoma (22). Thus, particular care is required when diagnosing primary thyroid LMS due to variant differential diagnoses.

The etiology of primary thyroid LMS remains unclear, however, certain authors have postulated that it may originate from the smooth muscle in the vascular walls $(6,10,14,16-18)$. In addition, LMS appears to invade adjacent tissue rather than metastasizing to regional lymph nodes $(6,11)$, thus, radical surgery is essential $(6,10,11,16,17,19)$. The complexity of the procedure depends on tumor size and adjacent tissue/organ involvement. Previous studies have shown that, in patients with a large or locally aggressive tumor, the more aggressive the procedure, the more beneficial it is $(18,19)$. However, aggressive surgical resection with adjuvant chemotherapy and radiation therapy have not been shown to affect recurrence rates or long-term survival of patients with primary thyroid LMS $(14,15)$. Of all previously published cases (1-19) and the present case, only the patient in the current study received immunotherapy. Certain studies suggested that immunotherapy has an effect on leimyosarcomas, however, the present case indicates that immunotherapy does not affect the outcome of primary thyroid LMS (23).

In conclusion, the present study reported a rare case of primary thyroid LMS in an 83-year-old male patient who underwent thyroid lobectomy and immunotherapy, but ultimately succumbed to the disease 5 months after undergoing a second surgery. Primary thyroid LMS is extremely rare and difficult to diagnose. Immunohistochemical staining is important for establishing a diagnosis of primary thyroid LMS and distinguishing the disease from anaplastic carcinoma. The standard primary therapy is radical surgery, as, to date, adjuvant chemotherapy, radiation therapy and immunotherapy have not proven beneficial. The long-term prognosis of primary thyroid LMS is poor. Therefore, careful evaluation of a patient's condition and comprehensive individual treatment are essential.

\section{References}

1. Adachi M, Wellmann KF and Garcia R: Metastatic leiomyosarcoma in brain and heart. J Pathol 98: 294-296, 1969.

2. Kawahara E, Nakanishi I, Terahata S and Ikegaki S: Leiomyosarcoma of the thyroid gland. A case report with a comparative study of five cases of anaplastic carcinoma. Cancer 62: 2558-2563, 1988.

3. Kaur A and Jayaram G: Thyroid tumors: Cytomorphology of medullary, clinically anaplastic and miscellaneous thyroid neoplasms. Diagn Cytopathol 6: 383-389, 1990.

4. Chetty R, Clark SP and Dowling JP: Leiomyosarcoma of the thyroid: Immunohistochemical and ultrastructural study. Pathology 25: 203-205, 1993

5. Iida Y, Katoh R, Yoshioka M, Oyama T and Kawaoi A: Primary leiomyosarcoma of the thyroid gland. Acta Pathol Jpn 43: 71-75, 1993.

6. Ozaki O, Sugino K, Mimura T, Ito K, Tamai S and Hosoda Y: Primary leiomyosarcoma of the thyroid gland. Surg Today 27: 177-180, 1997.

7. Thompson LD, Wenig BM, Adair CF, Shmookler BM and Heffess CS: Primary smooth muscle tumors of the thyroid gland. Cancer 79: 579-587, 1997.

8. Tsugawa K, Koyanagi N, Nakanishi K, Wada H, Tanoue K, Hashizume M and Sugimachi K: Leiomyosarcoma of the thyroid gland with rapid growth and tracheal obstruction: A partial thyroidectomy and tracheostomy using an ultrasonically activated scalpel can be safely performed with less bleeding. Eur J Med Res 4: 483-487, 1999.

9. Tulbah A, Al-Dayel F, Fawaz I and Rosai J: Epstein-Barr virus-associated leiomyosarcoma of the thyroid in a child with congenital immunodeficiency: A case report. Am J Surg Pathol 23: 473-476, 1999.

10. Takayama F, Takashima S, Matsuba H, Kobayashi S, Ito N and Sone S: MR imaging of primary leiomyosarcoma of the thyroid gland. Eur J Radiol 37: 36-41, 2001.

11. Day AS, Lou PJ, Lin WC and Chou CC: Over-expression of c-kit in a primary leiomyosarcoma of the thyroid gland. Eur Arch Otorhinolaryngol 264: 705-708, 2007.

12. Just PA, Guillevin R, Capron F, Le Charpentier M, Le Naour G, Menegaux F, Leenhardt L, Simon JM and Hoang C: An unusual clinical presentation of a rare tumor of the thyroid gland: Report on one case of leiomyosarcoma and review of literature. Ann Diagn Pathol 12: 50-56, 2008.

13. Mansouri H, Gaye M, Errihani H, Kettani F and Gueddari BE: Leiomyosarcoma of the thyroid gland. Acta Otolaryngol 128: 335-336, 2008.

14. Wang TS, Ocal IT, Oxley K and Sosa JA: Primary leiomyosarcoma of the thyroid gland. Thyroid 18: 425-428, 2008.

15. Bertelli AA, Massarollo LC, Volpi EM, Ueda RY and Barreto E: Thyroid gland primary leiomyosarcoma. Arq Bras Endocrinol Metabol 54: 326-330, 2010 (In Portuguese).

16. Amal B, El Fatemi H, Souaf I, Moumna K and Affaf A: A rare primary tumor of the thyroid gland: Report a new case of leiomyosarcoma and literature review. Diagn Pathol 8: 36, 2013.

17. Ege B and Leventoğlu S: Primary leiomyosarcoma of the thyroid. J Korean Surg Soc 85: 43-46, 2013.

18. Mouaqit O, Belkacem Z, Ifrine L, Mohsine R and Belkouchi A: A rare tumor of the thyroid gland: Report on one case of leiomyosarcoma and review of literature. Updates Surg 66: 165-167, 2014.

19. Tanboon J and Keskool P: Leiomyosarcoma: A rare tumor of the thyroid. Endocr Pathol 24: 136-143, 2013.

20. Bathan AJ, Constantinidou A, Pollack SM and Jones RL: Diagnosis, prognosis, and management of leiomyosarcoma: recognition of anatomic variants. Curr Opin Oncol 25: 384-389, 2013.

21. Agrawal M, Uppin SG, Challa S and Prayaga AK: Carcinosarcoma thyroid: An unusual morphology with a review of the literature. South Asian J Cancer 2: 226, 2013.

22. Naqiyah I, Zulkarnaen AN, Rohaizak M and Das S: Carcinosarcoma of the thyroid: A case report. Hippokratia 14: 141-142, 2010.

23. Edris B, Weiskopf K, Volkmer AK, Volkmer JP, Willingham SB, Contreras-Trujillo H, Liu J, Majeti R, West RB, Fletcher JA, et al: Antibody therapy targeting the CD47 protein is effective in a model of aggressive metastatic leiomyosarcoma. Proc Natl Acad Sci USA 109: 6656-6661, 2012. 